\title{
Intranasally delivered siRNA targeting PI3K/Akt/ mTOR inflammatory pathways protects from aspergillosis
}

\author{
P Bonifazi ${ }^{1}$, C D’Angelo ${ }^{1}$, S Zagarella ${ }^{1}$, T Zelante ${ }^{1}$, S Bozza ${ }^{1}$, A De Luca ${ }^{1}$, G Giovannini ${ }^{1}$, S Moretti ${ }^{1}$, \\ RG Iannitti $^{1}$, F Fallarino ${ }^{1}$, A Carvalho ${ }^{1}, \mathrm{C} \mathrm{Cunha}^{1}, \mathrm{~F}$ Bistoni $^{1}$ and L Romani $^{1}$
}

Innate responses combine with adaptive immunity to generate the most effective form of anti-Aspergillus immune resistance. Although some degree of inflammation is required for protection, progressive inflammation may worsen disease and ultimately prevents pathogen eradication. To define molecular pathways leading to or diverting from pathogenic inflammation in infection, we resorted to dendritic cells (DCs), known to activate distinct signaling pathways in response to pathogens. We found that distinct intracellular pathways mediated the sensing of conidia and hyphae by lung DCs in vitro, which translate in vivo in the activation of protective Th1/Treg responses by conidia or inflammatory Th2/Th17 responses by hyphae. In vivo targeting inflammatory (PI3K/Akt/mTOR) or anti-inflammatory (STAT3/IDO) DC pathways by intranasally delivered small interfering RNA (siRNA) accordingly modified inflammation and immunity to infection. Thus, the screening of signaling pathways in DCs through a systems biology approach may be exploited for the development of siRNA therapeutics to attenuate inflammation in respiratory fungal infections and diseases.

\section{INTRODUCTION}

Aspergillus spp. are ubiquitous in nature and the spectrum of diseases they cause is myriad, ranging from saprophytic colonization of pre-existing cavities (aspergilloma), allergic asthma, hypersensitivity pneumonitis, allergic bronchopulmonary aspergillosis occurring as a complication of bronchial asthma or cystic fibrosis, and disseminated disease associated with high mortality rate in patients with hematological malignancies and recipients of solid organs and stem cell transplants. Immunocompetent and non-atopic subjects are relatively resistant to infections and disease occurs in the setting of host damage. ${ }^{1,2}$

Current understanding of the pathophysiology underlying Aspergillus infection and disease highlights the molecular connection between the failure to resolve inflammation, lack of antifungal immune resistance, and susceptibility to Aspergillus infections and diseases. ${ }^{3,4}$ This condition is crucially exemplified in mice with chronic granulomatous diseases, in which an intrinsic, genetically determined failure to control inflammation to sterile fungal components determines the animals' inability to resolve an actual infection with Aspergillus fumigatus. ${ }^{4}$ In addi- tion, the association of persistent inflammation with intractable infection is common in non-neutropenic patients after allogeneic hematopoietic stem cell transplantation ${ }^{5}$ as well as in allergic fungal diseases. ${ }^{6}$ These observations highlight a truly bipolar nature of the inflammatory process in infection. Early inflammation prevents or limits infection, but an uncontrolled response may eventually oppose disease eradication. A main implication of these findings is that, at least in specific clinical settings, it is an exaggerated inflammatory response that likely compromises a patient's ability to eradicate infection, and not an 'intrinsic' susceptibility to infection that determines a state of chronic or intractable disease.

Dendritic cells (DCs) are central to the adaptive immunity and tolerance to Aspergillus. ${ }^{7}$ They sample the fungus in the lungs and then traffic to secondary lymphoid organs, where they communicate with lymphocytes to orchestrate adaptive immune responses. ${ }^{8,9}$ The capacity of DCs to initiate different adaptive immune responses to the fungus largely relied upon specialization and cooperation between distinct DC subsets ${ }^{10,11}$ and the discriminative recognition of fungal morphotypes by distinct innate recognition receptors. ${ }^{12-14}$ DC subsets in the lungs exert 
specific functions that can be associated with distinct expression of endocytic receptors, cell-surface molecules, and anatomical location within the lung. In homeostatic conditions, a fine-tuned balance exists between the various functions of lung DC subsets, which is necessary for maintaining immune homeostasis in the lung. However, infectious or inflammatory conditions can profoundly alter the functions of steady-state DC subsets and recruit inflammatory type DCs to the lung. This might be important for clearing the pathogenic stimulus, but could at the same time lead to immune pathology. ${ }^{15}$ In this regard, DCs expressing the enzyme indoleamine 2,3-dioxygenase (IDO), by means of activating regulatory $\mathrm{T}$ cells (Tregs), help to tame overzealous and exaggerated inflammatory responses in infection and allergy to the fungus ${ }^{11,16}$ and confer protective tolerance in hematopoietic transplantation. ${ }^{10}$ As a matter of fact, interleukin (IL)-23 produced by inflammatory DCs and the Th17 pathway, which downregulate tryptophan catabolism, favored pathology and served to accommodate the seemingly paradoxical association of chronic inflammation with fungal persistence. ${ }^{3,17}$ Thus, DCs, because of their heterogeneity and plasticity, are central in the early decision-making mechanisms that results in a given type of immune response to the fungus and determine the balance between immunopathology and protective immunity generated by the host-fungus interaction. ${ }^{7}$

In this study, we resorted to a systems biology analysis to define DC molecular pathways leading to or diverting from pathogenic inflammation in infection. We found that distinct intracellular pathways mediated the sensing of conidia and hyphae by lung- or bone marrow-derived DCs in vitro, which translate in vivo in the activation of protective Th1/Treg responses by conidia or inflammatory Th2/Th17 responses by hyphae. In vivo targeting inflammatory (PI3K/Akt/mTOR) or anti-inflammatory (STAT3/IDO) DC pathways by intranasally delivered small interfering RNA (siRNA) greatly impacted on inflammation and immunity to the fungus.

\section{RESULTS}

\section{DC subsets activate distinct antifungal Th/Treg cell responses in vivo}

We have already shown that bone marrow-derived inflammatory (i.e., granulocyte-macrophage (GM)-DCs) or tolerogenic (i.e., FLT3L-derived DCs, FL-DCs) DCs differently activate protective and non-protective antifungal responses in transplanted mice. ${ }^{10}$ We confirm here that GM-DCs pulsed with conidia marginally affected Th-cell activation, but pulsed with hyphae promoted the activation of Rorc + IL-17-producing and Gata3 + IL-4-producing CD $4+$ T cells with minimal activation of Tbet + IFN- $\gamma$-producing Th1 cells, and this resulted in limited control of fungal growth (Figure 1a and $\mathbf{b}$ ). In contrast, conidia-pulsed FL-DCs promoted the activation of Th1/Foxp3 + IL-10-producing T cells that accounted for the optimal control of fungal growth (Figure $\mathbf{1 a}$ and $\mathbf{b})$. Lung DCs, consisting of CD $11 \mathrm{c}^{\text {high }} / \mathrm{CD} 11 \mathrm{~b}^{\text {high }}$ cells (60-70\%) and CD11c+/CD8 + /B220+/CD103 + cells (20$30 \%),{ }^{15,18}$ recapitulated the results obtained with bone marrowderived DCs by showing that Th1/Treg protection was provided by DCs pulsed with conidia but not with hyphae (Figure 1a and $\mathbf{b}$ ). These data suggest that inflammatory DCs activate and regulatory DCs attenuate inflammatory responses to the fungus in the lungs.

\section{Distinct intracellular pathways are activated in DC subsets exposed to the fungus}

Activation of ERK/PI3K/Akt/mTOR pathways in response to hyphae. The activation of the intracellular kinases has a major role in DC function. ${ }^{19}$. Recent data also point to the emerging role of the PI3K/Akt/mammalian target of rapamycin (mTOR) pathway in DC regulation. ${ }^{20-23}$ Preliminary proteomic array approaches to simultaneously detect the relative level of phosphorylation of 19 kinases revealed that, $30 \mathrm{~min}$ after the exposure, Aspergillus hyphae more than conidia triggered the phosphorylation of ERK1/2, JNK, and PI3K/Akt/p70S6K in GM-DCs, whereas conidia more than hyphae triggered the phosphorylation of p38 and glycogen synthase kinase (GSK3) $\alpha / \beta$ in FL-DCs (Supplementary Figure 1). Further experiments in which the levels of kynase phosphorylation were assessed over time suggested that the activation of ERK1/2 (and JNK, data not shown) was prolonged ( $8 \mathrm{~h}$ ) and higher in GM-DCs than in FL-DCs, in response to hyphae, and similarly, p38 activation was sustained and higher in FL-DCs than in GM-DCs, in response to conidia (Figure 2a). Similar to bone marrow DCs, sustained ERK1/2 phosphorylation by hyphae (and similarly by swollen conidia, data not shown) and $\mathrm{p} 38$ phosphorylation by conidia were also observed in lung DCs (Figure 2a). Consistent with the finding that the dual signaling between MyD88 and TRIF is critical for gene expression programs and signaling networks in DCs, ${ }^{19}$ ERK activation was MyD88-dependent, whereas p38 phosphorylation was TRIF-dependent in lung DCs. In addition, the finding that either MAPK was oppositely regulated by MyD88 and TRIF in DCs, i.e, increased ERK activation in the absence of TRIF and p38 activation in the absence of MyD88, suggests the occurrence of reciprocal regulation between these two signaling pathways in DCs.

The two signaling pathways downstream of PI $3 \mathrm{~K}, \mathrm{mTOR}$ and GSK3, regulate cytokine production in DCs. ${ }^{20,23,24}$ The phosphorylation of Akt and p706SK kinases, that are downstream the mTOR pathway, ${ }^{21}$ induced by hyphae in GM-DCs prompted us to evaluate whether the mTOR complexes were activated by the fungus. Confirming the proteomic analysis, the mTORC2-dependent Ser ${ }^{473} \mathrm{Akt}$ was higher in response to hyphae than conidia in GM-DCs and lung DCs and was MyD88-dependent (Figure 2b).

Activation of noncanonical NF-KB/STAT3/IDO pathways in response to conidia. We next examined DC subsets for possible activation of canonical and noncanonical nuclear factor (NF) $-\kappa B$ transcription factors in response to the fungus. ${ }^{19}$ As the canonical pathway culminates in DNA binding of the classical NF$\kappa \mathrm{B}$ dimer $\mathrm{p} 50$-RelA and requires IKK $\beta$, and the noncanonical pathway involves the proteolytic processing of p100 to p52 and requires IKK $\alpha$, we measured IKK $\beta$ and IKK $\alpha$ phosphorylation in DC subsets exposed to the fungus. The two IkB kinase complex catalytic subunits have opposing roles, i.e., inflammation and immunity, with IKK $\alpha$ limiting the IKK $\beta$-dependent 

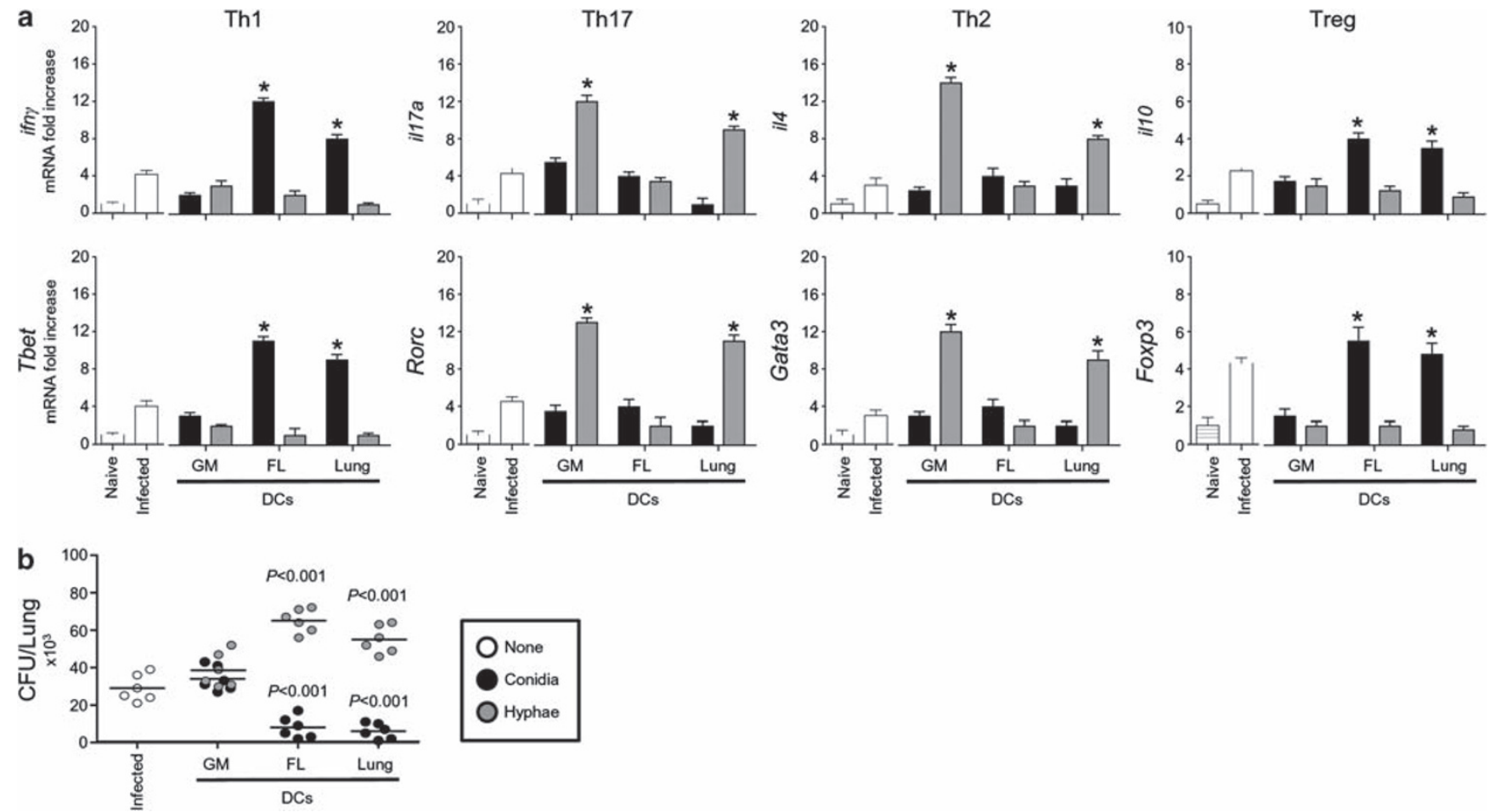

Figure 1 Dcendritic cell (DC) subsets activate distinct antifungal Th/Treg cell responses in vivo. C57BL6 mice received Aspergillus-pulsed DCs twice, a week apart, before the intranasal infection with the fungus. (a) Cytokine expression in the lungs and Th lineage-specific transcription factors expression in $\mathrm{CD}^{+} \mathrm{T}$ cells from thoracic lymph nodes were assessed by real-time PCR a week after the infection. (b) Fungal growth (colony-forming units, mean \pm s.e.). GM-CSF/IL-4-derived (GM) or FLT3L-derived (FL) DCs from bone marrow of C57BL6 and lung DCs, positively isolated from naive mice, were pulsed with live unopsonized conidia or hyphae for $2 \mathrm{~h}$ before adoptive transfer. ${ }^{\star} P<0.05$, DC-treated vs. untreated (Infected) mice. Representative of three experiments.

inflammatory pathway and leading to resolution of the early inflammatory process and development of Tregs. ${ }^{25}$ The phosphorylation of IKK $\beta$ but not IKK $\alpha$ was induced in GM-DCs and lung DCs exposed to hyphae, whereas the phosphorylation of IKK $\alpha$ occurred in FL-DCs and lung DCs in response to conidia (Figure 2c). Time course experiments revealed that the activation of the canonical/noncanonical NF- $\mathrm{\kappa B}$ progressively increased in response to hyphae or conidia, being still present at $180 \mathrm{~min}$ after the exposure (data not shown).

STAT3 is indispensable in FL-DCs development and ontogeny and conditions the tolerogenic state of DCs, thus acting as a negative regulator of immunostimulatory DCs. ${ }^{26}$ As STAT3 activates the noncanonical NF- $\mathrm{BB}$ pathway, as well as IDO, ${ }^{19}$ while inhibiting the canonical NF- $\mathrm{\kappa B}$ pathway, ${ }^{27}$ we assessed whether this would occur in response to the fungus and the impact on IDO activation. We measured $\mathrm{Tyr}^{705}$ phosphorylation of STAT3, which promotes its transcription activity, in DC subsets as well as the dependency on MyD88 and TRIF. STAT3 activation was not observed in GM-DCs after $2 \mathrm{~h}$ pulsing with hyphae, as opposed to FL-DCs or lung DCs pulsed with conidia, in which STAT3 phosphorylation occurred and was dependent on TRIF more than MyD88 (Figure 2d). However, STAT3 phosphorylation in GM-DCs was detected after $12 \mathrm{~h}$ stimulation with hyphae (data not shown). These data suggest that STAT3 activation occurred in FL-DCs more than GM-DCs through the TRIF pathway. The STAT3/noncanonical NF- $\kappa B$ pathway is required for the expression of Indo, ${ }^{16,28}$ one suppressive mechanism of
DCs. ${ }^{19,29,30}$ Confirming previous data ${ }^{16,19}$ and consistent with the IKK $\alpha$ expression, IDO protein expression occurred in FLDCs and lung DCs, but not in GM-DCs, in response to conidia in a p38/STAT3-dependent manner (Figure 2e). These data indicate that STAT3 phosphorylation in response to conidia is a proximal signaling for the activation of both noncanonical NF- $\kappa B$ and IDO in tolerogenic lung DCs.

To find out which pattern recognition receptor mediates the sensing of the different forms of the fungus upstream of MyD88 or TRIF, we assessed Akt and STAT3 phosphorylation in lung DCs exposed to Aspergillus hyphae or conidia, respectively, from several TLR- or Dectin-1-deficient mice. Akt phosphorylation was not observed in TLR2-deficient and, albeit partially, in Dectin-1-deficient DCs; similar to TRIF-deficient DCs, it was upregulated in TLR3-deficient DCs. STAT3 phosphorylation was not observed in TLR3- and, partially, TLR9- or Dectin1-deficient DCs. Both Akt and STAT3 phosphorylations were observed in DCs from TLR4-deficient mice (Figure 2f). Thus, Dectin-1, in association with TLR2, is involved in Akt activation in response to hyphae and, together with TLR3 and TLR9, in STAT3 phosphorylation in response to conidia.

The intracellular signaling pathways affect cytokine production. Although a comprehensive analysis of cytokine/chemokine production by DC subsets performed with the Bio-Plex cytokine assays is reported in Supplementary Figure 2, we addressed here the relative contribution of each signaling pathway on levels 


\section{ARTICLES}

a

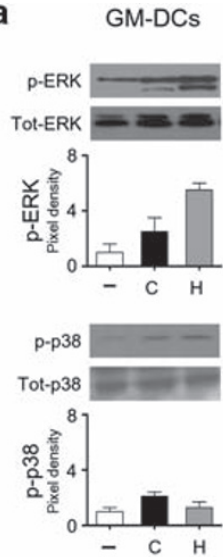

b
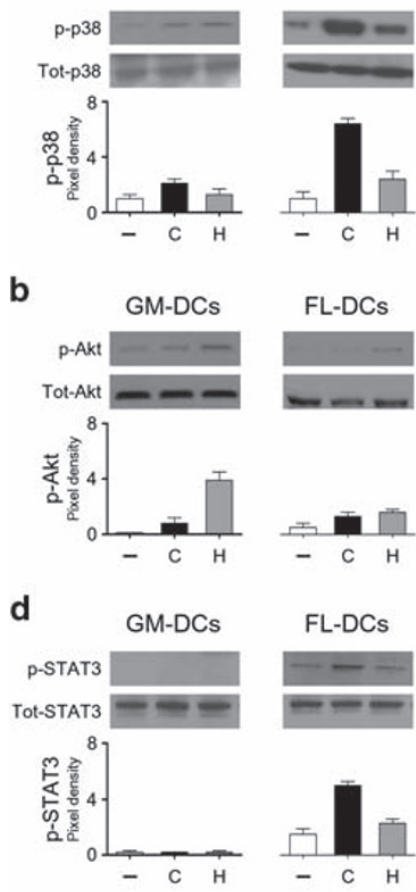

(1)
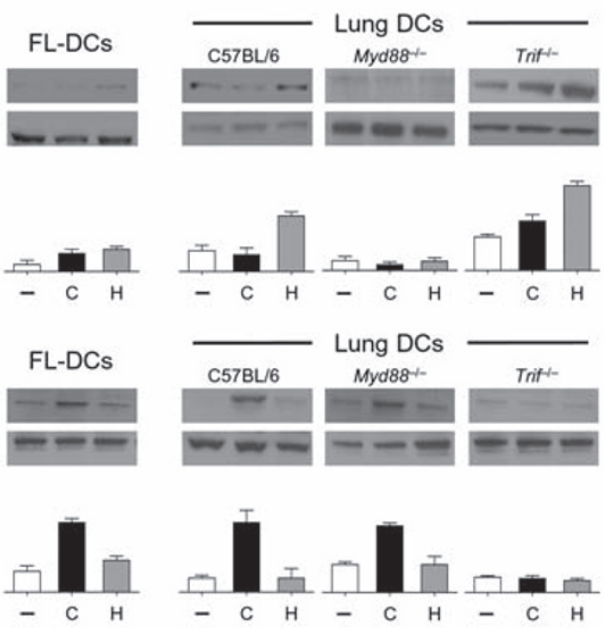

e
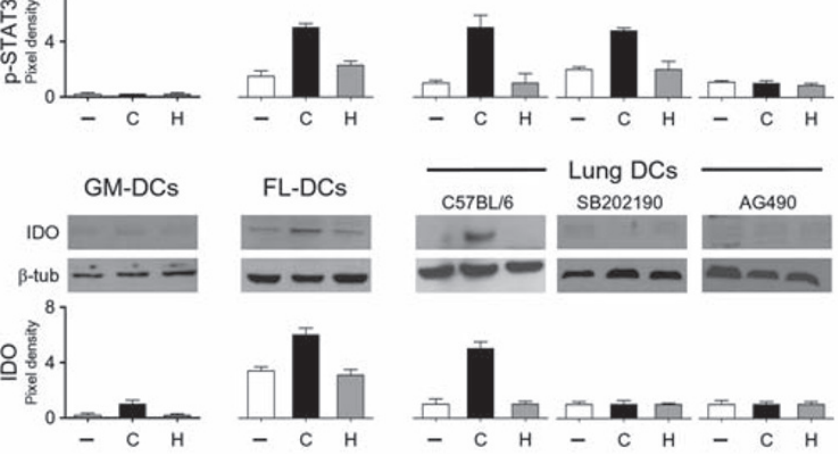

f
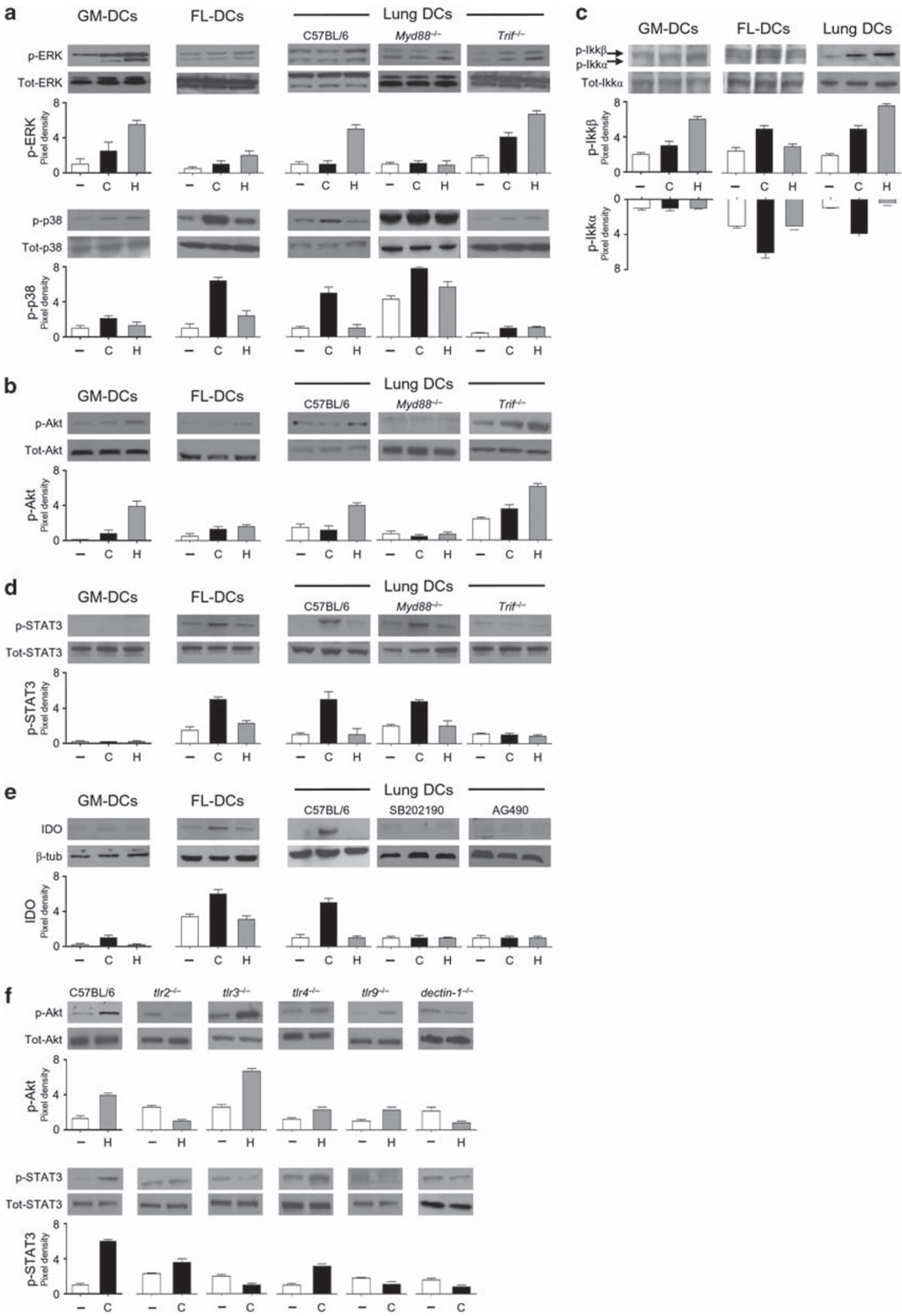

Tot-lkka

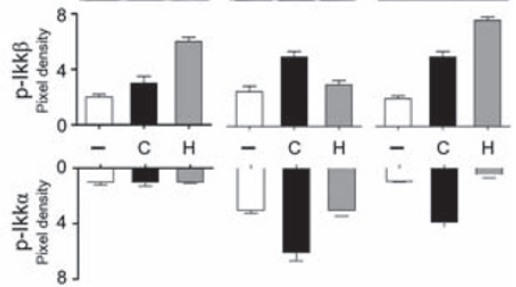

Figure 2 Activation of distinct intracellular pathways in dendritic cell (DC) subsets by Aspergillus fumigatus. Levels of (a) ERK1/2, p38 MAPK (8h), (b, f) Akt (8h), (c) IKK $\beta$ or IKK $\alpha$ (90 min), (d, f) STAT3 (2h), and (e) IDO (18h) phosphorylation with and without the presence of STAT3 (AG490, $25 \mu \mathrm{m}$ ) or p38 (SB202190, $5 \mu \mathrm{M}$ ) inhibitors (in e only), in GM-CSF/IL-4-derived (GM), FLT3L-derived (FL) DCs from bone marrow or purified lung CD11C+DCs from C57BL6, TLR- , Dectin-1, MyD88- or TRIF-deficient mice pulsed with live unopsonized conidia (black histograms), hyphae (gray histograms) or unpulsed (white histograms). Data are presented as immunoblots of cell lysates with phosphorylation-specific antibodies and fold increases (pixel density) in the phosphorylated to total protein ratios or to $\beta$-tubulin ( $\beta$-tub). Scanning densitometry was peformed on a Scion Image apparatus. Immunoblots were reblotted for $\beta$-actin to ensure equal protein loading. Representative of three experiments. 

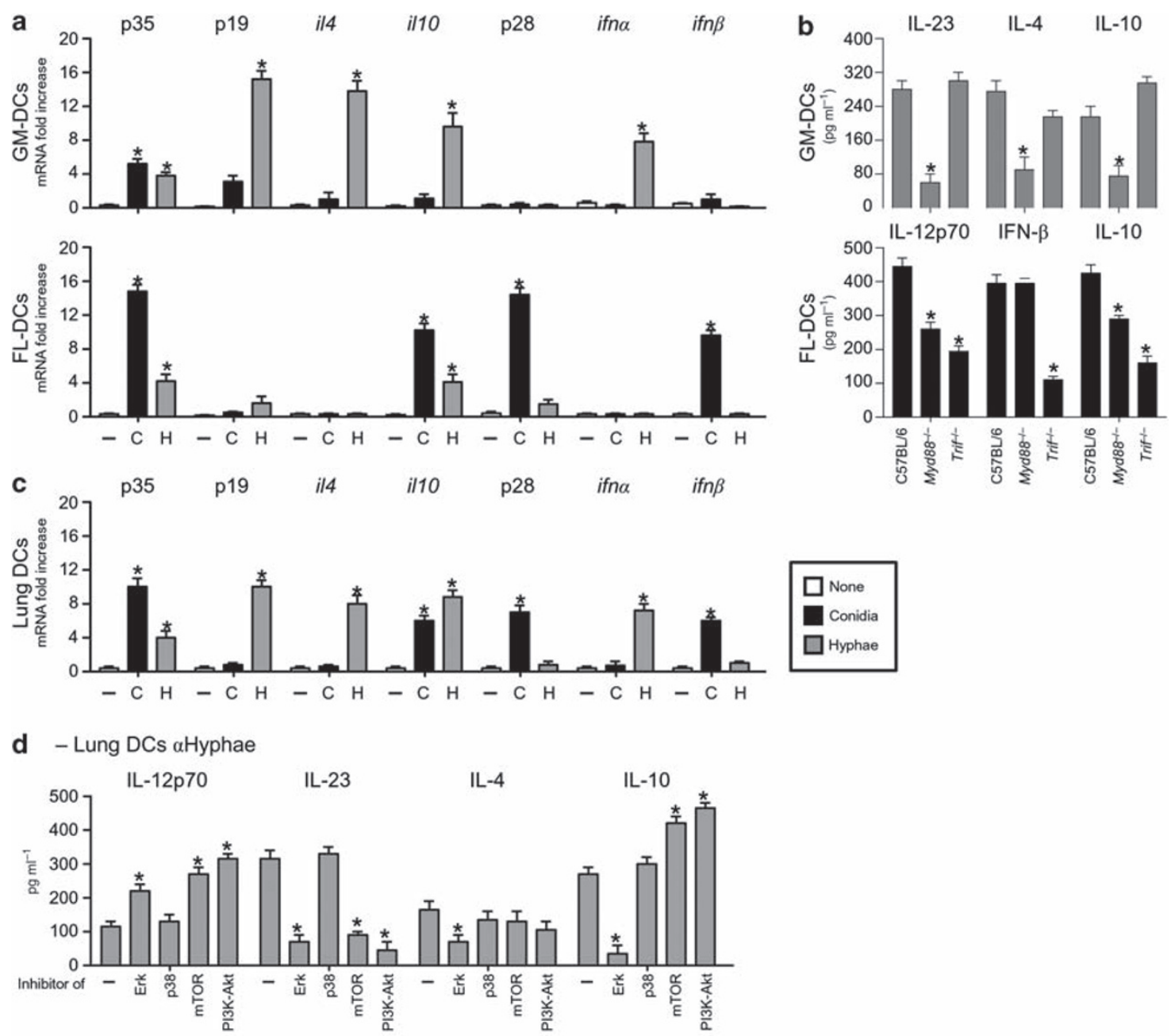

e - Lung DCs $\alpha$ Conidia

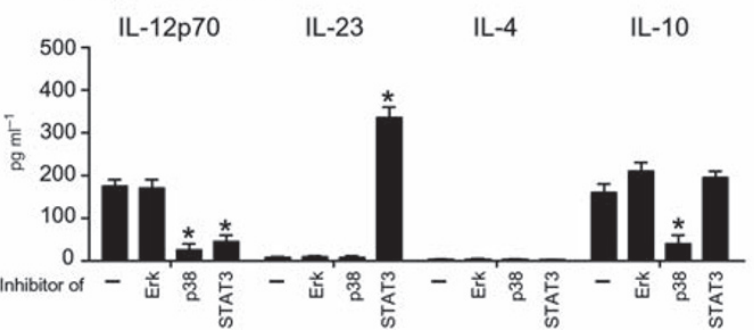

Figure 3 The intracellular signaling pathways affect cytokine production by dendritic cells (DCs) in response to Aspergillus. Cytokines (by real-time PCR, a, $\mathbf{c}$ or ELISA b, d, e) production by bone marrow-derived DC subsets or purified lung DCs from C57BL6, MyD88- or TRIF-deficient mice, unstimulated (-) or stimulated with conidia or hyphae (black and gray histograms, respectively) in the presence of inhibitors of ERK1/2 (15 $\mu \mathrm{M}, \mathrm{U} 0126)$,

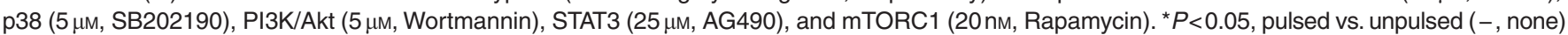
DCs; pulsed DCs with and without (-) inhibitors, and C57BL6 DCs vs. Myd88/- or Trif/- DCs. Representative of four experiments.

of IL-12p70, IL-23, IL-10, and IL-4 production, being the relative contribution of these cytokines to antifungal Th polarization well described. ${ }^{17,19}$ In addition, we also assessed the production of type I interferons (IFNs), given their role on IDO induction $^{30}$ and the anti-inflammatory IL-27 known to be produced in human DCs exposed to Aspergillus. ${ }^{31}$ Cytokines were assessed both in terms of mRNA expression and actual cytokine production by DCs in response to the different stimuli in the presence of inhibitors of ERK1/2, p38, PI3K/Akt, mTOR, and STAT3. p19, $i l 4$, and If $n$ - $\alpha$ mRNA expressions were high in GM-DCs or lung
DCs stimulated with hyphae, whereas p35, p28(IL-27), and Ifn- $\beta$ mRNA expressions were high in FL-DCs and lung DCs stimulated with conidia. Il10 mRNA expression was comparable in either DC subset stimulated with hyphae or conidia (Figure 3a and c). Cytokine production was MyD88 dependent in response to hyphae and mostly (IFN- $\beta$ ) TRIF- or (IL-12/IL-10) MyD88/ TRIF dependent in response to conidia (Figure $3 \mathbf{b}$ ).

Results with the inhibitors showed that cytokine production by lung DCs in response to hyphae was variably regulated by ERK, inhibiting IL-12 but promoting IL-23/IL-4/IL-10 and by 
PI3K/Akt/mTor, inhibiting IL-12/IL-10 but promoting IL-23 (Figure 3d). In response to conidia, the production of cytokines was largely independent from ERK but dependent on p38 and STAT3. Interestingly, inhibition of STAT3 promoted IL-23 production in response to conidia (Figure 3e), a finding confirming STAT3 at a nexus of signaling pathways regulating the inflammatory state of DCs in response to fungi. ${ }^{19}$ These data indicate that cytokine production by lung DCs implicates the inflammatory PI3K/Akt/mTOR pathway in response to hyphae or the tolerogenic p38/STAT3 pathway in response to conidia.

We have already shown that both murine and human DC subsets upregulated costimulatory molecules on exposure to the fungus. ${ }^{9}$ Consistent with the important role of the Notch ligands, Delta 4 and Jagged 1 and 2, in setting the outcome of immune reactivity, ${ }^{32}$ we found that Delta 4 and Jagged 2 expressions were upregulated in response to conidia, whereas expression of Jagged 1 was upregulated in response to hyphae (data not shown). As expression of Delta 4 in DCs is associated with the activation of IL-10-producing Th1 cells, whereas Jagged 1 and 2 expressions are variably involved in the induction of Th2/Treg cells, ${ }^{32}$ these data would predict that lung DCs are competent for the activation of distinct adaptive Th responses in vivo.

\section{Targeting DC inflammatory/anti-inflammatory pathways in vivo}

To assess whether targeting the PI3K/Akt/mTOR pathway in inflammatory DCs or the STAT3 anti-inflammatory pathways in tolerogenic DCs in vivo would impact on the severity of the infection, we resorted to different experimental approaches that include either the functional ablation of lung plasmacytoid (p)DCs endowed with tolerogenic, anti-inflammatory activity, ${ }^{18,33}$ or the selective targeting of the P13K/Akt/mTOR/STAT3 pathways by specific inhibitors or locally delivered siRNA.

Targeting by DCs. For the functional ablation of tolerogenic DCs, we injected mice with the pDCs depleting 120G8 antibody, known to selectively remove, although not completely, pDCs in the lung, 18,33 or adoptively transferred DCs with functional ablation of STAT3-by siRNA in vitro-or IDO-by using lung DCs from IDO-deficient mice. The treatment with the 120G8 antibody, given a day before and after Aspergillus infection, effectively reduced the number of $\mathrm{CD} 11 \mathrm{c}+\mathrm{B} 220+$ or $\mathrm{CD} 11 \mathrm{c}+$ Siglec $-\mathrm{H}+$ (a specific surface marker for $\mathrm{pDCs}$ in mice $^{34}$ ) cells in the lungs (Figure 4a) and resulted in increased neutrophil recruitment in the bronchoalveolar lavage (BAL) and lungs. Concomitantly, lungs of pDC-depleted mice showed enhanced inflammation and, partly, fungal growth (Figure $4 \mathbf{b}$ ). As anti-Gr-1 antibodies are also used to deplete DCs in the lungs, ${ }^{18,33}$ we injected mice with antiGrl antibodies. The treatment significantly reduced the number of CD11c +CD11b + cells (from 60 to $38 \%$ on total lung cells, data not shown) but not that of B220 + or Siglec-H + CD11c + cells as assessed by flow cytometry on gated CD11c + DCs enriched from lungs (Figure 4a). Thus, residual Gr-1- $1^{-}$DCs are still present after treatment. Depletion of CD11c+CD11b + cells and neutrophils, as observed in the BAL and lungs (Figure $4 \mathrm{~b}$ ), likely accounted for the extensive fungal growth in the relative absence of inflammatory cell recruitment in the lung (Figure $\mathbf{4 b}$ ).
On assessing the impact of either treatment on antiinflammatory/inflammatory pathway activation in vivo, we first noticed that, similar to the in vitro findings, infection was associated with increased Akt, more than STAT3, phosphorylation in the lung, and that Akt phosphorylation occurred in response to swollen conidia and STAT3 phosphorylation in response to resting conidia (Figure 4c). Importantly, Akt phosphorylation occurred in purified lung CD11c+CD11b + cells and STAT3 phosphorylation in purified CD11c+B220 + pDCs (Figure 4d), a finding suggesting that distinct $\mathrm{DC}$ subsets in vivo account for the heterogenous responses of lung DCs to the different fungal forms. Accordingly, pDC depletion resulted in decreased phosporylation of STAT3, but not Akt, whereas Gr-1 + cell depletion resulted in decreased Akt phosphorylation, without affecting STAT3 phosphorylation (Figure 4e). Experiments with adoptively transferred STAT3-deficient, by siRNA in vitro, or IDO-deficient conidia-pulsed DCs directly proved that the STAT3/IDO axis curbs the inflammatory response in infection, as shown by the increased expression of inflammatory cytokines (IL-23p19 and il17a), decreased expression of IL-12p35/il10, and the relative failure to control fungal growth (Figure $4 \mathrm{f}$ ). The finding that STAT3 or IDO deficiency in hyphae-pulsed DCs did not apparently affect the control of fungal growth (Figure 4f), further points to the crucial regulatory function of the STAT3/IDO axis in modulating inflammation and immunity to conidia.

Targeting by agonists/antagonists. Experiments with STAT3 antagonists/agonists complemented the above findings by showing that treatment with the STAT3 antagonist, JSI- $124,{ }^{19}$ greatly impaired resistance to infection, as indicated by the decreased survival and increased fungal growth, neutrophil recruitment, and inflammatory lung pathology (Figure 5a). The levels of IL-23p19 and $i l 17 a$ were higher and those of IL-12p35/il10 lower in the lungs of JSI-124-treated than in untreated mice, and this paralleled the high (Rorc) and low (Foxp3) levels mRNA expression in TLN CD4 + T cells (Figure 5b and $\mathbf{c}$ ). Interestingly, deregulated immunity following JSI-124 treatment included susceptibility to allergy, as indicated by the increased Th2 cytokines (IL-4/IL-5/IL-13), eosinophils number, and tissue damage in experimental fungal allergy (Supplementary Figure 3). In contrast, the STAT3- ${ }^{35}$ and Foxp $3+$ Treg $-{ }^{36}$ inducer FLT3L greatly increased resistance to infection, as indicated by the survival and the reduced fungal growth, neutrophil recruitment and lung inflammation (Figure 5a), low levels of IL-23p19, $i l 17 a$ (in the lung) and Rorc + T cells (in TLN) and high levels of IL-12p35 and $i l 10$ (in the lung ), and Foxp3 + Treg (in TLN) (Figure 5b). These data suggest that STAT3 is central in balancing the inflammatory/anti-inflammatory state of DCs, and both the innate and adaptive antifungal immunity are deregulated in condition of STAT3 deficiency, as already demonstrated. ${ }^{19}$

To define the possible contribution of the PI3K/Akt/mTOR pathway to the deregulated inflammation and immune homeostasis caused by STAT3 deficiency, mice were treated with the inhibitors of PI3K (wortmannin, targeting the p110 catalytic subunit of PI3K), Akt (perifosine, inhibiting Akt membrane translocation), or mTOR (rapamycin, inhibiting mTORC1). ${ }^{37}$ 
To avoid possible direct antifungal effects, the inhibitors were administered for 3 consecutive days before the injection of conidia 2 days after the last treatment. Treatments with wortmannin and perifosine reduced the fungal growth and the expression of inflammatory chemokines $(\mathrm{cxcl} 2)$ and cytokines (IL-23p19), while increasing the IL-12p35/il10 expression in the lungs (Figure 5c) and promoting Th1/Treg cell activation in the TLN (Figure 5d). The ability of perifosine also to inhibit

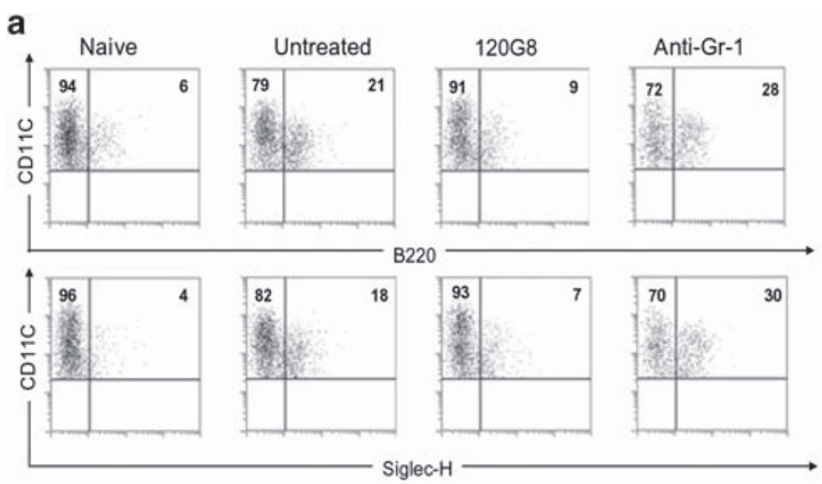

b Untreated

$120 \mathrm{G} 8$

Anti-Gr-1
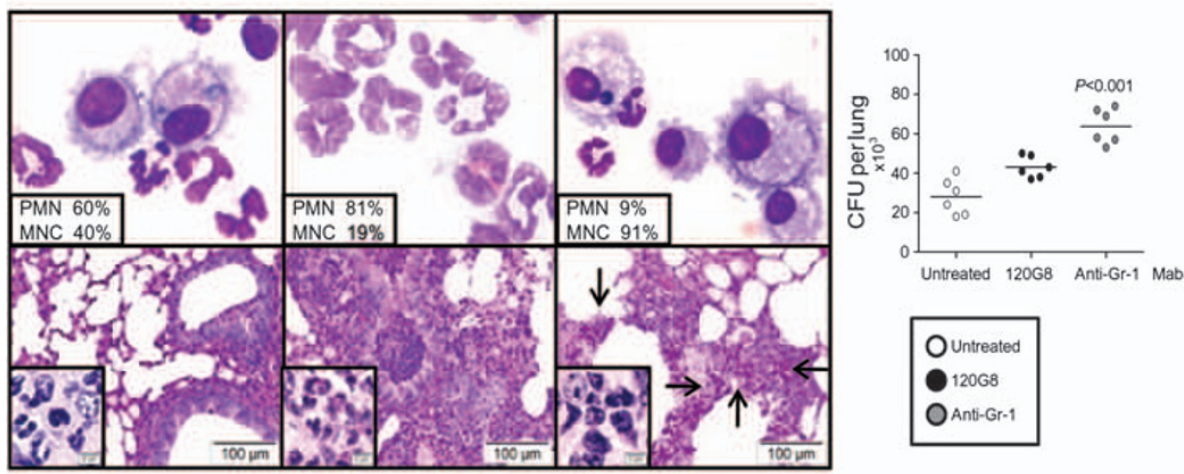

C
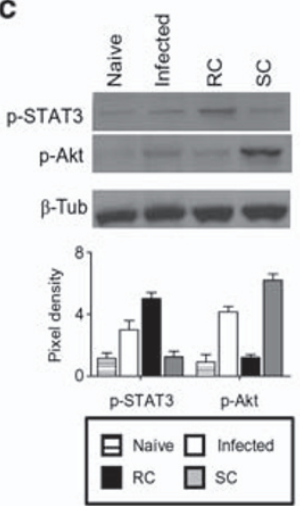

d
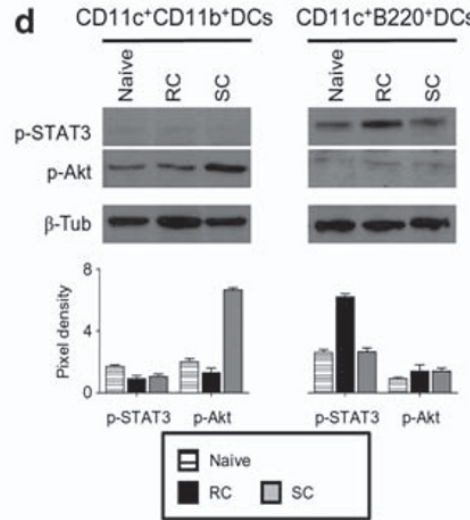

e
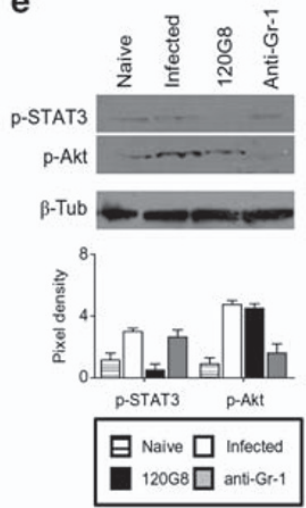

Figure 4 Targeting inflammatory/anti-inflammatory pathways in vivo with dendritic cells (DCs). Mice were intranasally infected with Aspergillus conidia and treated with the pDCs depleting 120G8 or anti-Gr-1 Ab (clone RB6-8C5) 1 day before and day 1 after Aspergillus infection. Control mice received an isotype control antibody. (a) Flow cytometry of gated CD11c+DCs enriched from lungs 3 days after the infection. Numbers refer to percent positive cells. (b) Bronchoalveolar lavage (BAL) morphometry, lung histology (periodic acid-Schiff staining) and fungal growth (colony-forming unit (CFU), mean \pm s.e.) were assessed 3 days after the infection. Total and differential cell counts were done by staining BAL smears with May-Grünwald Giemsa reagents. Numbers refer to \% polymorphonuclear (PMN) or mononuclear (MNC) cells. Photographs were taken using a high-resolution Microscopy Olympus DP71. Note the limited neutrophil recruitment in the BAL and lungs (magnified in the inset) and the unrestricted fungal growth (arrows) in anti-Gr-1-treated mice, as opposed to the apparent neutrophil recruitment in the BAL and lungs (magnified in the inset) of 120G8-treated mice. Bars indicated magnifications. Representative of two experiments. $P$, treated vs. untreated mice. STAT3 and Akt phosphorylation in the lungs (c) or purified lung DC subsets (d) from C57BL6 mice intranasally infected with live conidia or inactivated resting (RC) or swollen (SC) conidia a day before and (e) in the lungs of mice treated with the $120 \mathrm{G} 8$ or anti-Gr-1 MAb. STAT3 and Akt phosporylation are expressed as immunoblots of cell lysates with phosphorylation-specific antibodies and fold increases (pixel density) in the phosphorylated to $\beta$-tubulin ( $\beta$-tub). (f) C57BL6 mice received conidia or hyphae-pulsed DCs from C57BL6 (WT) or IDO-deficient mice twice, a week apart, before the intranasal infection with the fungus. DCs were STAT3depleted by siRNA in vitro before adoptive transfer. Cytokine expression in the lungs by real time PCR and fungal growth (CFU, mean \pm s.e.) were assessed 3 days after the infection. ${ }^{*} P<0.05$, DC-treated vs. untreated (infected) mice. Representative of two experiments. 
f
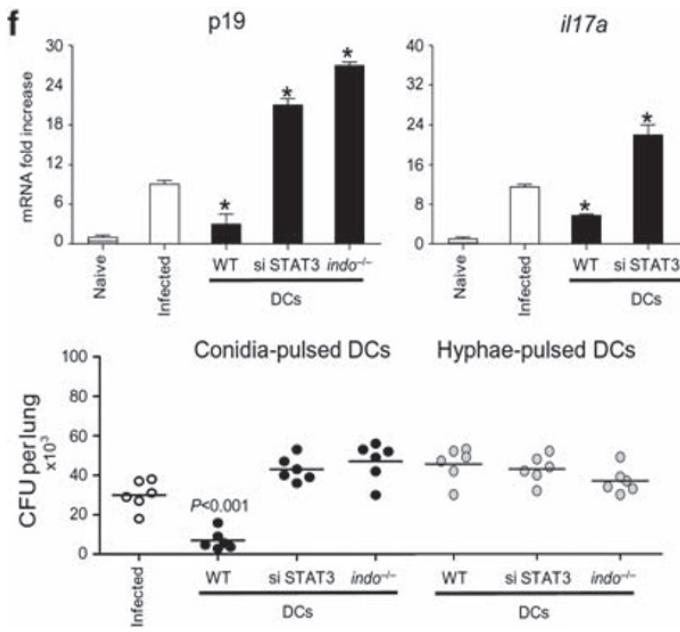

il17a

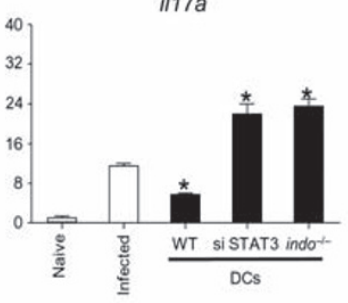

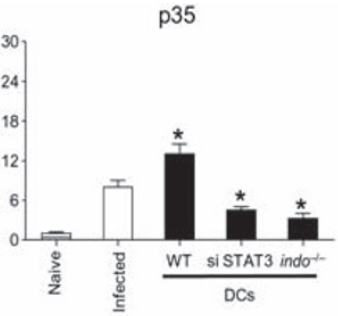

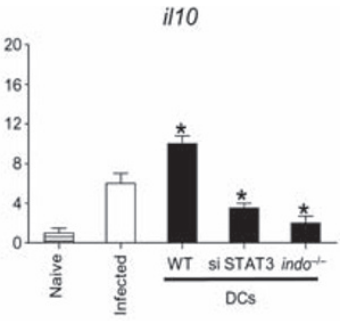

Figure 4 Continued.

the ERK/JNK activation ${ }^{38}$ may explain the apparently superior activity of perifosine over wortmannin. Interestingly, not only both treatments did not cause lung inflammation, as indicated by the low number of neutrophil recrutiment, but, as expected, concomitant treatment with perifosine significantly reduced lung inflammation in JSI-124-treated mice (Figure 5e), a finding suggesting the contribution of the PI3K/Akt axis to inflammation in condition of STAT3 deficiency. Consistent with the concurrent Akt activation following rapamycin treatment, ${ }^{21}$ rapamycin failed to restrict the fungal growth and to decrease inflammatory cytokine production and inflammatory neutrophil recruitment in the lungs (Figure 5c), a finding in line with the proinflammatory side effects observed after rapamycin treatment to ameliorate chronic allograft damage. ${ }^{37}$ Together, these data indicate that targeting selective anti-inflammatory pathways in vivo ameliorates the infection process and immunity to it.

Targeting by siRNA. Modulation of gene expression has become the new promise in the development of therapeutics against many serious diseases. To assess whether targeting murine inflammatory/anti-inflammatory gene in vivo would similarly impact on the infection, we resorted to siRNA inhalation, a route of siRNA delivery successfully used to treat asthma and other pulmonary diseases. ${ }^{39}$.We silenced STAT3 and, due to the existence of PI3K and Akt highly homologous isoforms, ${ }^{40} \mathrm{mTOR}$ to block downstream Ser ${ }^{47} \mathrm{Akt}$ phosphorylation. Mice received siRNA twice 2 days before infection and were assessed for the parameters of inflammation and infection, 3 days after the infection. The results show that, similar to STAT3 blockade, si STAT3 reduced the activation of STAT3 in the lung (Figure 6a), increased the fungal growth (Figure 6c), the inflammatory cell influx in the BAL and lungs and tissue pathology (Figure 6d). All these parameters were instead greatly ameliorated, including a better control of fungal growth, on si mTOR administration that effectively decreased Akt phosphorylation and mTOR protein expression (Figure 6a, $\mathbf{c}$ and $\mathbf{d}$ ). The failure of control siRNA to affect these parameters indicated the limited occur- rence of unintended stimulation of the innate immune system or off-target effects upon local administration of siRNA in vivo. Importantly, both siRNAs affected signal-transduction pathways in purified lung CD11c + DCs (Figure $6 \mathbf{b}$ ), a finding suggesting that DCs are targeted by siRNA in vivo, although the contribution of other cells cannot be excluded. As a matter of fact, Akt phosphorylation on si mTOR was also decreased on lung macrophages (data not shown). Together, these data suggest that targeting anti-inflammatory pathways in vivo protects from aspergillosis, and siRNAs could be exploited as therapeutic agents in aspergillosis.

\section{DISCUSSION}

This study shows that DC signaling pathways in response to Aspergillus could be successfully exploited to prevent pathogenic inflammation and deregulated infection (Figure 7). As Aspergillus, through the subversion of host inflammatory pathways, ${ }^{41}$ may promote inflammatory responses, an inflammatory vicious circle is likely at work in infection, the targeting of which may offer strategies to control or prevent exacerbation of Aspergillus infection and disease. Confirming the inflammatory role of murine and human DCs in response to hyphae, ${ }^{8-10}$ ERK and PI3K/Akt conditioned bone marrow-derived and lung conventional CD11b + DCs for inflammatory Th2/Th17 priming, as predicted by the production of IL-4, IL-23, and IL- $1 \beta$, which are cytokines required for Th2 and Th $17^{8,42}$ activation in response to the fungus. Recent data indicate an emerging role of the $\mathrm{PI} 3 \mathrm{~K} / \mathrm{mTOR}$ pathway as a key integrator of activating and inhibitory pathways involved in DC differentiation and activation. ${ }^{20,22,24}$ We found that mTOR activation occurred in GM-DCs and lung CD11b + DCs in response to hyphae and contributed to inflammation in vitro and in vivo, despite the fact that the MTOR inhibitor, rapamycin, also promoted inflammation. Given the emerging complex activity of mTOR kinase signaling in inflammation but also on effector/Treg lineage commitment, ${ }^{43-45}$ much remains to be elucidated regarding mTOR activity in aspergillosis. 
a
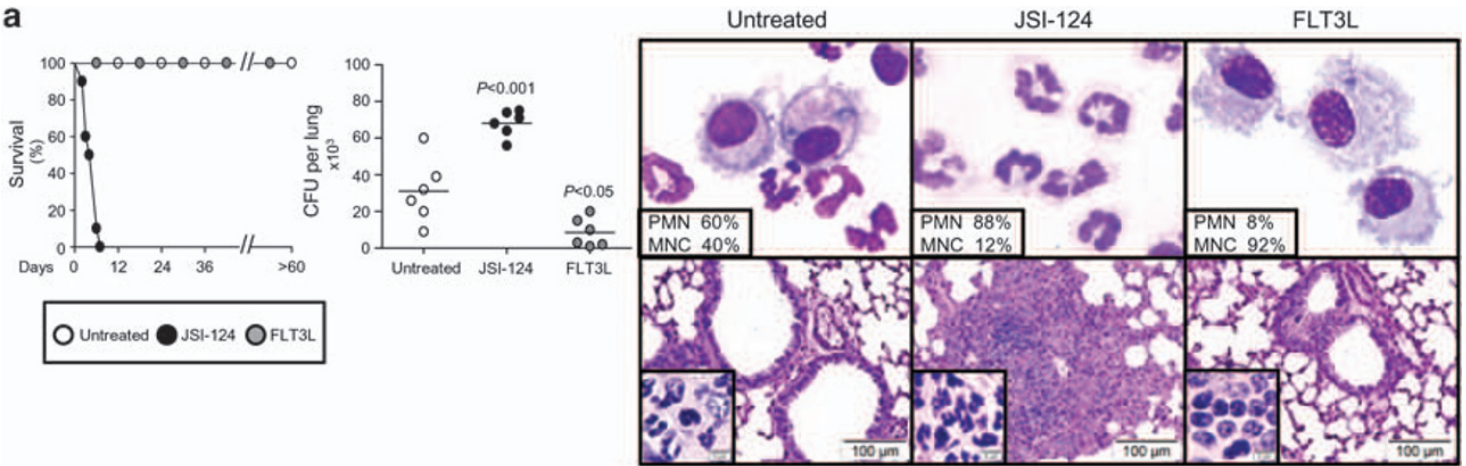

b
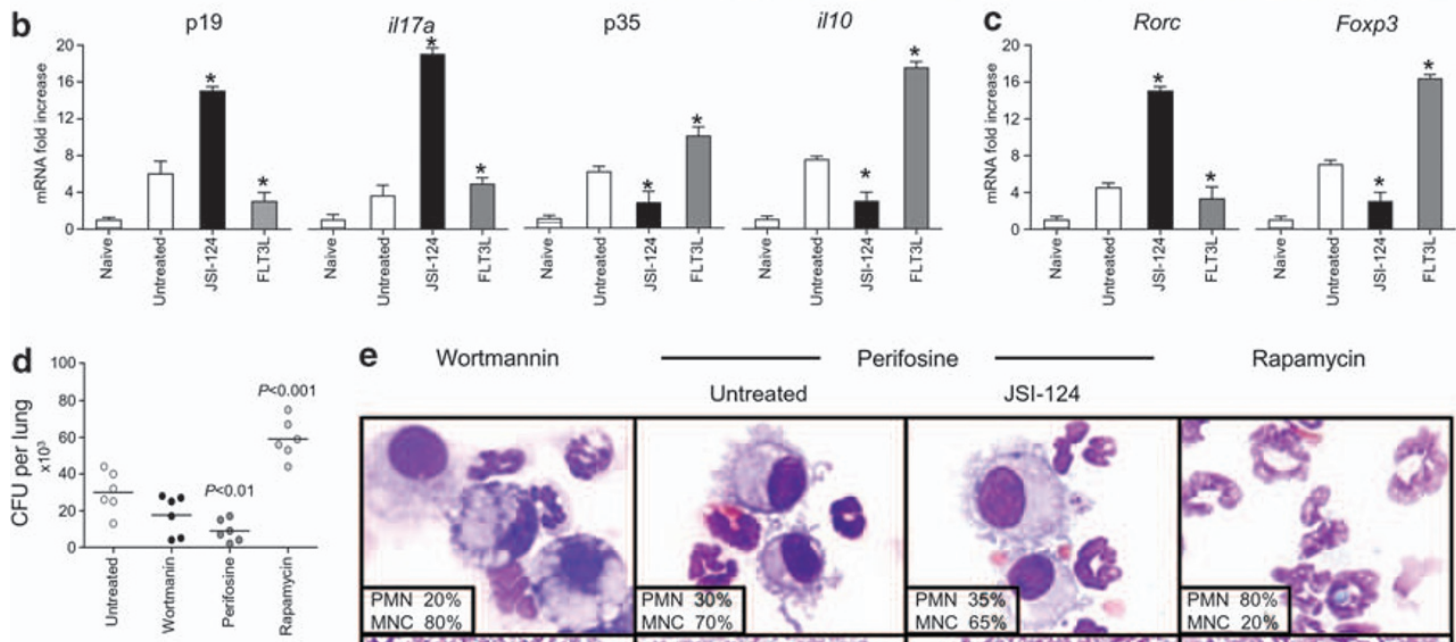

e Wortmannin
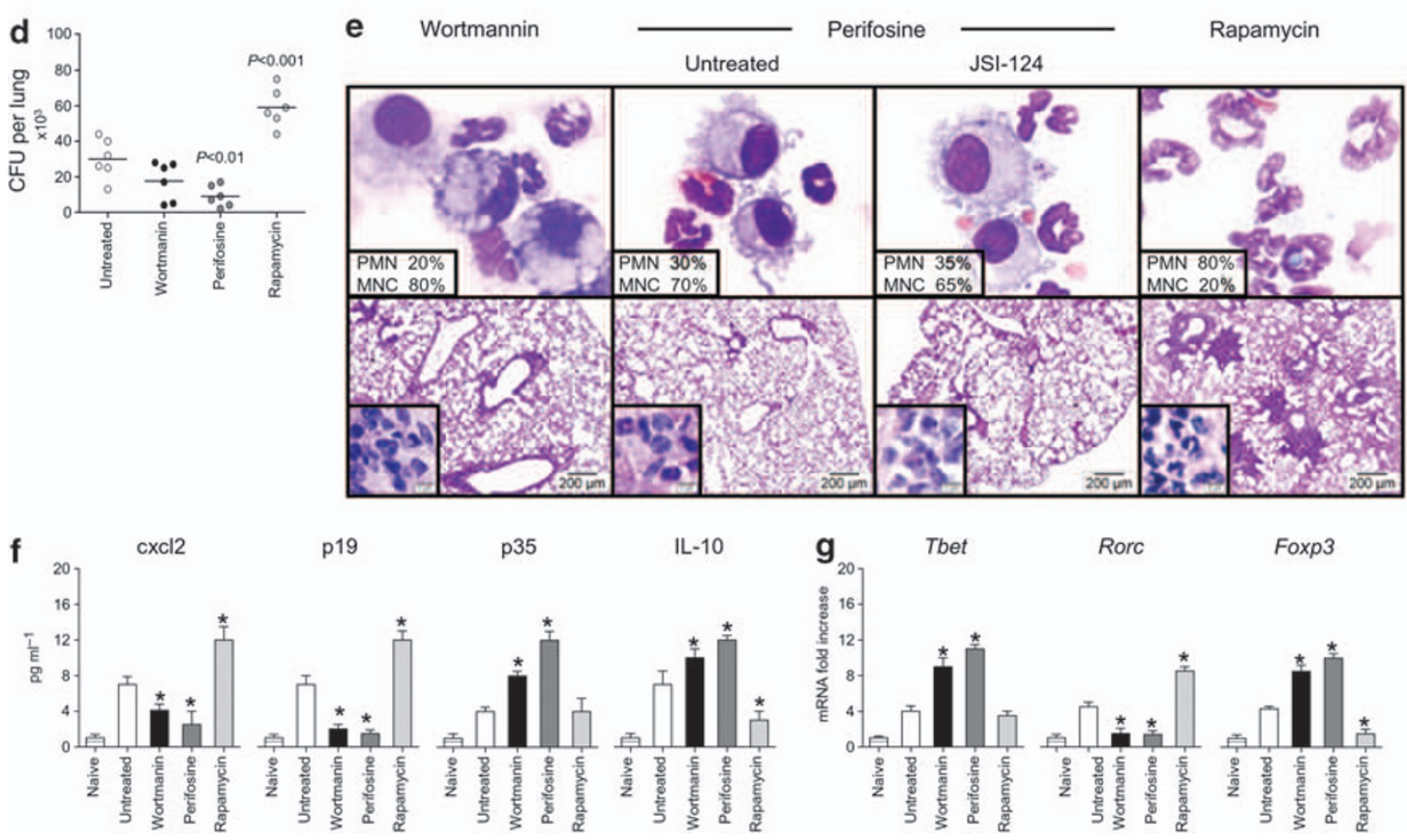

Foxp3

Figure 5 Targeting DC inflammatory/anti-inflammatory pathways in vivo by specific inhibitors. Mice were treated intraperitoneally for 3 consecutive days, followed by 2 days of resting before the intranasal infection, with $1 \mathrm{mg} \mathrm{kg}^{-1} \mathrm{JSI}-124$ or $0.5 \mathrm{mg} \mathrm{kg}^{-1} \mathrm{FLT} 3 \mathrm{~L}$ and assessed for (a) survival, fungal growth (colony-forming units, mean \pm s.e.), bronchoalveolar lavage (BAL) morphometry (May-Grünwald Giemsa staining), lung histology (periodic acid-Schiff (PAS) staining), (b) cytokine expression in the lung or (c) Th specific transcripts in CD4+T cells from TLN (real-time PCR) 3 days after the infection. Control mice received dimethylsulfoxide. Total and differential cell counts were performed as in legend to Figure 4. Note the reduced neutrophil recruitment in BAL and lungs (magnified in the inset) and amelioration of lung pathology in mice treated with FLT3L as opposed to the severe influx of neutrophils and tissue pathology seen in JSI-124-treated mice. $P$, treated vs. untreated mice. Representative of three experiments. (d) Mice infected as above were treated with $1 \mathrm{mg} \mathrm{kg}^{-1}$ Wortmannin, $36 \mathrm{mg} \mathrm{kg}^{-1}$ Perifosine, or $10 \mathrm{mg} \mathrm{kg}^{-1}$ Rapamycin and assessed for fungal growth, (e) BAL morphometry and lung histology (PAS staining), (f) cytokine expression in the lung and (g) Th specific transcripts in CD4+T cells from TLN (real-time PCR) 3 days after the infection. Note the limited neutrophil recruitment in BAL and lungs (magnified in the inset) and lung pathology in mice treated with Wortmannin or Perifosine alone as opposed to Rapamycin-treated mice and the amelioration of lung inflammatory pathology and neutrophil recruitment in lungs (magnified in the inset) in JSI-124-treated mice concomitantly treated with perifosine (e). ${ }^{*} P<0.05$, treated vs. untreated mice.

Confirming the pivotal role of the p38 pathway in IL12 p70 production and DC maturation, ${ }^{19}$ a sustained $\mathrm{p} 38$, TRIF-dependent, pathway favored a Th1/Treg outcome in FL-DCs or lung pDCs stimulated with conidia. As a matter of fact, the production of cytokines associated with tolerogenic DCs, namely IL-10, IFN- $\beta$, and IL-27, was strictly p38/TRIFdependent. These findings are consistent with the notion of a differential requirement for TRIF/MyD88 signaling pathways 
in the activation of DCs, ${ }^{19}$ the relative independency of antiAspergillus Th1 responses from the MyD88 pathway ${ }^{14,46}$ and the involvement of TLR ${ }^{14,47}$ and C-type lectin receptors $^{12,13,48}$ upstream of MyD88 in the inflammatory response to Aspergillus. Accordingly, TLR2 and Dectin-1 mediate the activation of inflammatory Akt, whereas TLR3 and, partially, TLR9 and Dectin-1, mediate STAT3 activation. Both TLR9 ${ }^{16}$ and
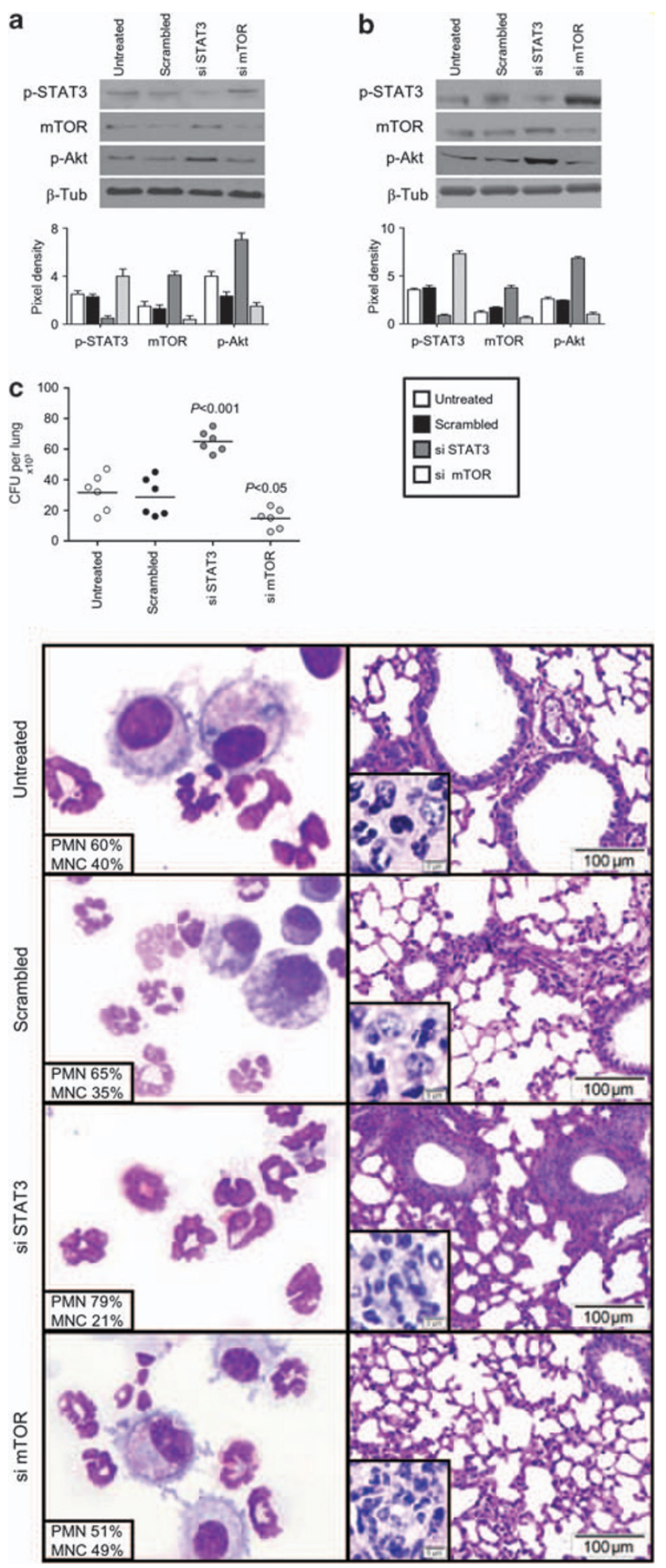

Dectin- $1^{49}$ are known to deliver activating and tolerogenic signals to DCs.

Confirming previous data with Candida albicans, ${ }^{19}$ STAT3 signaling played a nonredundant role in the tolerogenic/ anti-inflammatory state of DCs in vitro and in vivo, such that both the innate and adaptive antifungal immunity were deregulated in condition of STAT3 deficiency. STAT3 activation promptly occurred in FL-DCs and lung pDCs through TRIF and $\mathrm{p} 38$. It is of interest that GSK3, known to favor the nucleocytoplasmic shuttling of STAT3, ${ }^{50}$ is activated by conidia more than hyphae. Consistent with the finding that tolerogenic DCs are required for inhibition of immunopathology and pathogen clearance, ${ }^{33}$ functional deficiency of STAT3 impaired the ability of conidia-pulsed DCs to confer protection in infection. The finding that STAT3 blockade resulted in IL-23 production provides evidence for the contribution of this pathway to the interconvertibility between DC subsets, thus further adding to the flexibility and plasticity of DC responses in vivo. However, the late STAT3 expression in hypha-stimulated GM-DCs suggests a possible two-step model of activation, in which the late activation of STAT3/IL-10 by proinflammatory stimuli may serve to limit the activation state of DCs. ${ }^{19}$

Tryptophan catabolism is known to contribute to the tolerogenic state of DCs in infection and allergy to the fungus. ${ }^{4,16,30}$ We had already shown that conidia-pulsed tolerogenic FL-DCs induced tolerizing responses to Aspergillus by a mechanism involving IDO. ${ }^{10,11}$ Similar to FL-DCs, we found that IDO activation occurred in lung DCs on exposure to conidia through noncanonical NF- $\mathrm{KB}$ activation and STAT3, and was associated with the induction of protective tolerance in infection.

The implications of our findings are manifold. Not only could these findings be useful in the design of fungal vaccines capable of targeting the appropriate signaling pathways in DCs, but screening of signaling pathways in DCs through a systems biology approach may be exploited for the development of therapeutics that target inflammation in aspergillosis. Although these signaling pathways may likely affect functions of cells other than DCs, this study establishes a proof-of-principle that the selective targeting of inflammatory/anti-inflammatory DC pathways in vivo greatly impacted in the susceptibility and severity of the infection. By targeting the most proximal P13K

Figure 6 Targeting dendritic cell (DC) inflammatory/anti-inflammatory pathways in vivo by small interfering RNA (siRNA). C57BL6 were treated twice, 2 days before the intranasal conidia infection, with $10 \mathrm{mg} \mathrm{kg}^{-1}$ of unmodified siRNA or equivalent doses of nonspecific control siRNA duplex in a volume of $20 \mu \mathrm{l}$ of TransITR-EE Starter Kit. STAT3, Akt phosphorylation or mTOR protein expression (immunoblots of lung lysates with phosphorylation-specific antibodies and fold increases (pixel density) in the phosphorylated to total protein ratios or to $\beta$-tubulin ( $\beta$-tub) in lung cells (a) or purified CD11c+DCs (b), fungal growth (c), BAL morphometry (May-Grünwald Giemsa staining), lung histology (periodic acid-Schiff staining) and inflammatory cell recruitment (magnified in the inset) (d) were carried out 3 days after the infection. Total and differential cell counts were performed as in legend to Figure 4. Bars indicated magnifications. $P$, treated vs. untreated mice. Shown are the results pooled from two experiments (three animals per group). 


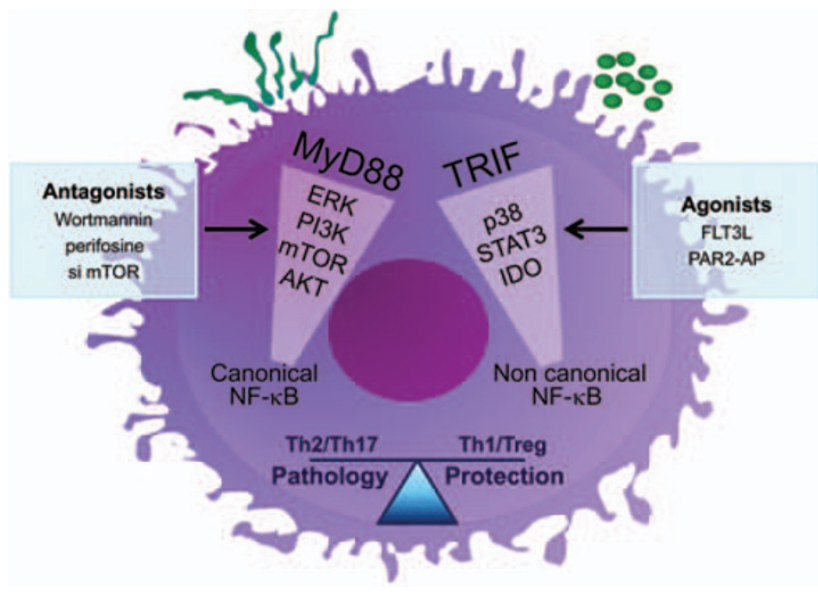

Figure 7 Targeting inflammatory pathways in aspergillosis through a systems biology approach. The figure shows that the activation of distinct signaling pathways in dendritic cells (DCs) translates recognition of the fungus into distinct inflammatory and adaptive immune responses. The screening of signaling pathways in DCs through a systems biology approach may be exploited for the development of small interfering RNA (siRNA) therapeutics to attenuate inflammation in respiratory fungal infections and diseases. In boxes, targets of possible anti-inflammatory strategies. IDO, 2,3 indoleamine dioxygenase, PAR2-AP, ${ }^{41}$ protease-activated receptor 2 agonist peptide; $\mathrm{Si}$, small interference. See text for details.

or the more distal Akt/mTOR pathways by specific inhibitors, we found that inhibition of the inflammatory Akt pathway by perifosine or wortmannin resulted in the control of the infection and prevented deregulated inflammation in condition of STAT3 blockade. However, although useful to unravel the cellular consequences of pathway inhibition, the therapeutic use of these inhibitors is hampered by their high toxicity. ${ }^{37}$

The intranasal administration of siRNA has opened new avenues in drug delivery and respiratory therapy. ${ }^{39,51}$ Although the in vivo use of siRNA has been limited, they have huge potential as therapeutic agent given the absence of adverse immune reactions and systemic access. ${ }^{52}$ Adding to the potential of siRNA as therapeutic agents in the areas of cancer and viral infections, our study is the first to show the potential of siRNA to attenuate inflammation in respiratory fungal infections. Although the precise mechanisms by which siRNA works in vivo is a major focus of our future studies, the degree of protection matched the anti-inflammatory activity of the siRNA in cell culture, further points to the use of the DC systems biology ${ }^{53}$ approach for quick screening of siRNA for therapeutics.

Ultimately, because immunity is neither simply a set of discrete signaling pathways activated by pathogens nor a function of the host, the use of systems biology approaches ${ }^{54}$ proved to be useful for the generation of new therapeutics that targets pathogenicity rather than microbial growth, the host-pathogen interface rather than the pathogen and promotes protective immune responses.

\section{METHODS}

\section{Mice}

Female C57BL6 mice, 8- to 10-weeks old, mice were purchased from Charles River (Calco, Italy). Homozygous Tlr $2^{-/-}, \mathrm{Tlr}^{-{ }^{--}}, \mathrm{Tlr}^{-4^{--}}, \mathrm{Tlr}^{-9^{--}}$, $\mathrm{Myd}_{88^{-/-}}$, Trif ${ }^{-/}$, Indo $^{-/-}$(Indotm $\left.1 \mathrm{Alm} / \mathrm{J}\right)$, and Dectin-1-deficient mice on a C57BL6 background were bred under specific pathogen-free conditions at the Animal Facility of Perugia University, Perugia, Italy. Experiments were performed according to the Italian Approved Animal Welfare Assurance A-3143-01.

\section{Fungal strains, infections, allergy, and treatments}

The strain of A. fumigatus was obtained from a fatal case of pulmonary aspergillosis at the Infectious Diseases Institute of the University of Perugia. Viable conidia ( $>95 \%$ ) were obtained by growth on Sabouraud dextrose agar (Difco Laboratories, Detroit, MI) supplemented with chloramphenicol for 4 days at room temperature. Inactivated resting or swollen conidia were obtained as described..$^{8,55}$ For hyphae, $10 \times 10^{4}$ conidia were incubated in Sabouraud broth at $28^{\circ} \mathrm{C}$ and harvested $12 \mathrm{~h}$ later, at the time at which between 80 and $85 \%$ of single conidia had germinated with a germ-tube emergence between 9 and $30 \mu$ in length. For infection, mice were anesthetized by intraperitoneal (i.p.) injection of $2.5 \%$ avertin (Sigma Chemical, St Louis, MO) before instillation of a suspension of $2 \times 10^{7}$ conidia/ $20 \mu \mathrm{l}$ saline intranasally. Fungi were suspended in endotoxin-free (Detoxi-gel; Pierce, Rockford, IL) solutions ( $<1.0 \mathrm{EU} \mathrm{ml}^{-1}$, as determined by the LAL method). Mice were monitored for survival (\% surviving mice) and fungal growth (colony-forming units/organ, mean \pm s.e.). ${ }^{11}$ Mice dying of fungal challenge routinely underwent necropsy for histopathological confirmation of pulmonary aspergillosis. BAL was performed by cannulating the trachea and washing the airways with $3 \mathrm{ml}$ of phosphate-buffer saline to collect the BAL fluid. Total and differential cell counts were performed by staining BAL smears with May-Grünwald Giemsa reagents (Sigma) before analysis. At least 200 cells per cytospin preparation were counted and the absolute number of each cell type was calculated. Photographs were taken using a high-resolution Microscopy Olympus DP71 (Olympus, Milan, Italy). For histology, lung tissue sections were stained using periodic acid-Schiff. Mice were treated i.p. for 3 consecutive days, followed by 2 days of resting before the infection, as follows: $1 \mathrm{mg} \mathrm{kg}^{-1}$ JSI-124 (cucurbitacin I, Tocris Bioscience, Bristol, UK), $0.5 \mathrm{mg} \mathrm{ml}^{-1}$ FLT3L (R\&D Systems, Milan, Italy), $1 \mathrm{mg} \mathrm{kg}^{-1}$ wortmannin (Calbiochem, San Diego, CA), $36 \mathrm{mg} \mathrm{kg}^{-1}$ perifosine, a synthetic alkylphospholipid (LoPiccolo, 2008 \#91) (Cayman Chemical, AA, MI) and $10 \mathrm{mg} \mathrm{kg}^{-1}$ rapamycin (Tocris Bioscience). Preliminary dose per effect experiments indicated that the efficacy of wortmannin occurred within the range of $0.1-10 \mathrm{mg} \mathrm{kg}^{-1}$, of perifosine within the range of $3.6-36 \mathrm{mg} \mathrm{kg}^{-1}$, and of rapamycin within $1-10 \mathrm{mg} \mathrm{kg}^{-1}$. Control received dimethylsulfoxide. For DC depletion, mice received $150 \mu$ g per i.p. of $120 \mathrm{G} 8$ rat IGg2a MAb ${ }^{56}$ or $100 \mu$ g per i.p anti-Gr-1 rat IGg2b MAb ${ }^{57}$ (clone RB6-8C5) 1 day before and day 1 after Aspergillus infection. Control mice received an isotype control antibody (rat IgG; SigmaAldrich, Milano, Italy).

\section{Cell preparation and culture}

GM-DCs or FL-DCs were obtained by culturing bone marrow cells with $150 \mathrm{U} \mathrm{ml}^{-1}$ mouse rGM-CSF (Sigma) $+75 \mathrm{U} \mathrm{ml}^{-1} \mathrm{rIL}^{-4}$ (R\&D Systems) in Iscove medium supplemented with glutamine, penicillin. and streptomycin, and $10 \%$ heat-inactivated fetal calf serum for 7 days or $200 \mathrm{ng} \mathrm{ml}^{-1}$ FLT3-L for 9 days. ${ }^{19}$. Purified lung CD11 $\mathrm{c}^{+}$DCs were further separated in B220 + or CD11b + cells by magnetic cell sorting with MicroBeads (Miltenyi Biotec, Bologna, Italy) as described. ${ }^{11}$ Inhibitors of ERK1/2

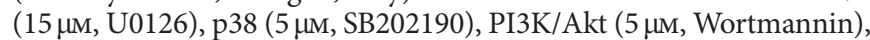
STAT3 (25 $\mu \mathrm{M}$, AG490), and mTORC1 (20 nm, Rapamycin, Tocris Bioscience) were purchased from Calbiochem and dissolved at $1000 \times$ the final concentration in dimethylsulfoxide (Sigma). DCs were pre-exposed to the inhibitors for $120 \mathrm{~min}$ at $37^{\circ} \mathrm{C}$ before stimulation. Control cells were treated with an identical amount of dimethylsulfoxide.

\section{Flow cytometry}

All staining reactions were performed at $4^{\circ} \mathrm{C}$. Cells were first incubated with $2.4 \mathrm{G} 2 \mathrm{Fc}$ receptor Ab to reduce nonspecific binding. Dead cells and debris were excluded using propidium iodide. Staining with fluorescein isothiocyanate-labeled anti-B220 (RA3-6B2, BD PharMingen, Buccinasco, Italy) and Siglec-H (HM1075F, HyCult biotechnology b.v., 
SPACE Import Export s.r.l, Milan, Italy). Abs was carried out on gated $\mathrm{CD} 11 \mathrm{c}+\mathrm{DCs}$ purified from lungs. Cells were analyzed with a FACScan flow cytofluorometer equipped with C ELLQuestTM software (Becton Dickinson, Mountain View, CA).

\section{Adoptive transfer of DCs, fungal challenge, and assessment of protection}

After $2 \mathrm{~h}$ of pulsing, DCs $\left(5 \times 10^{5}\right)$ were administered i.p. in $20 \mu \mathrm{l}$ phosphate-buffered saline twice, at weekly interval, before the infection. Mice were assessed for fungal growth and Th/Treg cell activation by reverse transcription-PCR on purified (Miltenij Biotec) $\mathrm{CD}^{+}(>98 \%) \mathrm{T}$ cells from draining thoracic lymph nodes, a week later.

\section{Western blotting}

Protein phosphorylations were assessed on DCs stimulated as above. Blots of cells lysates were incubated with rabbit polyclonal Abs recognizing the unphosphorylated form of ERK, p38, Akt, STAT3 or phospho-p44/p42 ERK (Thr202/Tyr294), phospho-p38 (Thr180/tyr182), phospho-Akt (Ser 473), and phospho-STAT3 (Tyr705) followed by horseradish peroxidase-conjugated anti-mouse or anti-rabbit IgG, as per the manufacturer's instruction Cell Signaling Technology (Euroclone, Milan, Italy). Rabbit mTOR antibody (2972) was from Cell Signaling Technology. Blots were developed with the enhanced chemiluminescence detection kit (Amersham Pharmacia Biotech, Milan, Italy). Immunoblotting for IDO was performed with rabbit polyclonal IDO-specific antibody on DCs after 18-h stimulation. ${ }^{19}$ The positive control consisted of IDO-expressing $\mathrm{MC}_{24}$ transfectants and the negative control of mock-transfected $\mathrm{MC}_{22}$ cells. Scanning densitometry was performed on a Scion Image apparatus, Scion Corporation, Maryland. The pixel density of bands was normalized against total proteins or $\beta$-tubulin.

\section{Canonical and noncanonical NF- $\kappa$ B}

Anti-phospho-IKK $\alpha$ (Ser180)/IKK $\beta$ (Ser181) rabbit Abs (Cell Signaling Technology) were used for western blotting of phospho IKK $\alpha$ and IKK $\beta$.

\section{siRNA design}

The Accel siRNA SMART-pool duplexes of predesigned mouseSTAT3 siRNA (STAT3, catalog number E-040794-00) were from Dharmacon (Chicago, IL). The siRNA sequences specific for mouse stat3 (sense, 5' -ACACUACAAACAUCAGAACCAAGCC-3'; antisense, 5' -GAUGUGAUGUUUGUAGUCUUUGGUUCGG-3') or mTOR (sense, 5' -ACAUCGAAGCUAGGACUAAGCCCAG-3' ${ }^{\prime}$; antisense, 5' -GGUGUAUCUUCGAUCCUGAUUCGGGUC-3') were selected, synthesized, and annealed by the manufacturer, and were used in combination with negative control siRNA (Ambion, Monza, Italy). Nonspecific siRNA scrambled duplex were synthesized by IDT (Tema Ricerca Srl, Bologna, Italy).

\section{Delivery of siRNA duplexes in vitro and in vivo}

Dendritic cells were seeded into 96-well plate and then added of siRNA in a final concentration of $1 \mu \mathrm{M}$ for $72 \mathrm{~h}$ according to the manufacturer's instructions (Dharmacon). The silencing effects of siRNAs were confirmed by western blotting. For in vivo studies, each mouse was lightly anesthetized by inhaled diethyl ether then given intranasal, unmodified siRNA $\left(10 \mathrm{mg} \mathrm{kg}^{-1}\right)$ or equivalent doses of nonspecific control siRNA duplex in a volume of $20 \mu \mathrm{l}$, in the TransIT ${ }^{\mathrm{R}}$-EE Starter Kit (Tema Ricerca Srl). It is known that lung-specific siRNA delivery can be achieved by intranasal administration without the need for viral vectors or transfection agents in vivo. ${ }^{58}$ Intranasal siRNA was given twice 2 days before infection.

\section{ELISA and reverse transcriptase-PCR and real-time PCR}

The level of cytokines in culture supernatants $(24 \mathrm{~h})$ of DCs were determined by Kit ELISA (R\&D System). The detection limits of the assays were $<10$ for IL-12p70, and $<3$ for IL-10 and IL-4, $<30$ for IL-23 and $<7$ for IFN- $\beta$. Real-time reverse transcription-PCR was performed using the iCycler iQ detection system (Bio-Rad Life Science Group, Segrate, Italy) and SYBR Green chemistry (Finnzymes Oy, Espoo, Finland). Cells were lysed and total RNA was extracted using RNeasy Mini Kit (QIAGEN, Milan, Italy) and was reverse transcribed with Sensiscript Reverse Transcriptase (QIAGEN), according to the manufacturer's directions. The PCR primers were as described. ${ }^{19}$ Amplification efficiencies were validated and normalized against Gapdh. The thermal profile for SYBR Green real time PCR was at $95^{\circ} \mathrm{C}$ for $3 \mathrm{~min}$, followed by 40 cycles of denaturation for $30 \mathrm{~s}$ at $95^{\circ} \mathrm{C}$ and an annealing/extension step of $30 \mathrm{~s}$ at $60^{\circ} \mathrm{C}$. Each data point was examined for integrity by analysis of the amplification plot. The mRNA-normalized data were expressed as relative cytokine mRNA in stimulated cells compared with that of mock-infected cells.

\section{Statistical analysis}

Data were analyzed by GraphPad Prism 4.03. program (GraphPad Software, San Diego, CA). Student's $t$-test or analysis of variance and Bonferroni's test were used to determine the statistical significance $(P)$ of differences in organ clearance and in vitro assays. The data reported are either from one representative experiment out of three to five independent experiments (western blotting and reverse transcription-PCR) or pooled from three to five experiments, otherwise. The in vivo groups consisted of six to eight mice per group.

SUPPLEMENTARY MATERIAL is linked to the online version of the paper at http://www.nature.com/mi

\section{ACKNOWLEDGMENTS}

We thank Dr Cristina Massi Benedetti for digital art and editing. The original studies conducted in the author laboratory were supported by the Specific Targeted Research Project "MANASP" (LSHE-CT-2006), contract number 037899 (FP6), by the Italian Projects PRIN 2007KLCKP8_004 (to LR), 2007XYB9T9 001 (to SB) and 2007KLCKP8 005 (to FB), and by the Project 2006.020.0291 from Fondazione Cassa di Risparmio di Perugia.

\section{DISCLOSURE}

The authors declared no conflict of interest.

(C) 2010 Society for Mucosal Immunology

\section{REFERENCES}

1. Marr, K.A., Patterson, T. \& Denning, D. Aspergillosis. Pathogenesis, clinical manifestations, and therapy. Infect. Dis. Clin. North Am. 16, 875894 vi (2002).

2. Denning, D.W., O'Driscoll, B.R., Hogaboam, C.M., Bowyer, P. \& Niven, R.M. The link between fungi and severe asthma: a summary of the evidence. Eur. Respir. J. 27, 615-626 (2006).

3. Romani, L., Zelante, T., De Luca, A., Fallarino, F. \& Puccetti, P. IL-17 and therapeutic kynurenines in pathogenic inflammation to fungi. J. Immunol. 180, 5157-5162 (2008).

4. Romani, L. et al. Defective tryptophan catabolism underlies inflammation in mouse chronic granulomatous disease. Nature 451, 211-215 (2008).

5. Ortega, M. et al. Prospective evaluation of procalcitonin in adults with nonneutropenic fever after allogeneic hematopoietic stem cell transplantation. Bone Marrow Transplant 37, 499-502 (2006).

6. Schubert, M.S. Allergic fungal sinusitis. Clin. Rev. Allergy Immunol. 30, 205-216 (2006).

7. Romani, L. in Aspergillus and Aspergillosis (eds. Latgè, J. P. \& Steinbach, W.J.) 247-261 (ASM Press: Washington D.C., USA, 2008).

8. Bozza, S. et al. Dendritic cells transport conidia and hyphae of Aspergillus fumigatus from the airways to the draining lymph nodes and initiate disparate Th responses to the fungus. J. Immunol. 168, 1362-1371 (2002).

9. Bozza, S. et al. A dendritic cell vaccine against invasive aspergillosis in allogeneic hematopoietic transplantation. Blood 102, 3807-3814 (2003).

10. Romani, L. et al. Thymosin alpha1 activates dendritic cell tryptophan catabolism and establishes a regulatory environment for balance of inflammation and tolerance. Blood 108, 2265-2274 (2006).

11. Montagnoli, C. et al. Immunity and tolerance to Aspergillus involve functionally distinct regulatory $T$ cells and tryptophan catabolism. J. Immunol. 176, 1712-1723 (2006). 
12. Mezger, M. et al. Proinflammatory response of immature human dendritic cells is mediated by dectin-1 after exposure to Aspergillus fumigatus germ tubes. J. Infect. Dis. 197, 924-931 (2008).

13. Serrano-Gomez, D. et al. Dendritic cell-specific intercellular adhesion molecule 3-grabbing nonintegrin mediates binding and internalization of Aspergillus fumigatus conidia by dendritic cells and macrophages. J Immunol 173, 5635-5643 (2004).

14. Bellocchio, S. et al. The contribution of the Toll-like/IL-1 receptor superfamily to innate and adaptive immunity to fungal pathogens in vivo. J. Immunol. 172, 3059-3069 (2004).

15. GeurtsvanKessel, C.H. \& Lambrecht, B.N. Division of labor between dendritic cell subsets of the lung. Mucosal. Immunol. 1, 442-450 (2008).

16. Grohmann, U. et al. Reverse signaling through GITR ligand enables dexamethasone to activate IDO in allergy. Nat. Med. 13, 579-586 (2007).

17. Zelante, T. et al. IL-23 and the Th17 pathway promote inflammation and impair antifungal immune resistance. Eur. J. Immunol. 37, 2695-2706 (2007).

18. van Rijt, L.S. et al. In vivo depletion of lung CD11C+ dendritic cells during allergen challenge abrogates the characteristic features of asthma. J. Exp. Med. 201, 981-991 (2005).

19. Bonifazi, P. et al. Balancing inflammation and tolerance in vivo through dendritic cells by the commensal Candida albicans. Mucosal. Immunol. 2, 362-374 (2009).

20. Weichhart, T. et al. The TSC-mTOR signaling pathway regulates the innate inflammatory response. Immunity 29, 565-577 (2008).

21. Weichhart, T. \& Saemann, M.D. The multiple facets of mTOR in immunity. Trends Immunol. 30, 218-226 (2009).

22. Fukao, T. et al. PI3K-mediated negative feedback regulation of IL-12 production in DCs. Nat. Immunol. 3, 875-881 (2002).

23. Martin, M., Rehani, K., Jope, R.S. \& Michalek, S.M. Toll-like receptormediated cytokine production is differentially regulated by glycogen synthase kinase 3. Nat. Immunol. 6, 777-784 (2005).

24. Cao, W. et al. Toll-like receptor-mediated induction of type I interferon in plasmacytoid dendritic cells requires the rapamycin-sensitive $\mathrm{PI}(3) \mathrm{K}$ mTOR-p70S6K pathway. Nat. Immunol. 9, 1157-1164 (2008).

25. Bonizzi, G. \& Karin, M. The two NF-kappaB activation pathways and their role in innate and adaptive immunity. Trends Immunol. 25, 280-288 (2004).

26. Barton, B.E. STAT3: a potential therapeutic target in dendritic cells for the induction of transplant tolerance. Expert Opin. Ther Targets 10, 459-470 (2006).

27. Nadiminty, N. et al. Stat3 activation of NF-\{kappa\}B p100 processing involves CBP/p300-mediated acetylation. Proc. Natl. Acad. Sci. USA 103, 7264-7269 (2006).

28. Tas, S.W. et al. Noncanonical NF-kappaB signaling in dendritic cells is required for indoleamine 2,3-dioxygenase (IDO) induction and immune regulation. Blood 110, 1540-1549 (2007).

29. Mellor, A.L. \& Munn, D.H. IDO expression by dendritic cells: tolerance and tryptophan catabolism. Nat. Rev. Immunol. 4, 762-774 (2004).

30. Puccetti, P. \& Grohmann, U. IDO and regulatory T cells: a role for reverse signalling and non-canonical NF-kappaB activation. Nat. Rev. Immunol. 7, 817-823 (2007).

31. Gafa, V. et al. Human dendritic cells following Aspergillus fumigatus infection express the CCR7 receptor and a differential pattern of interleukin-12 (IL-12), IL-23, and IL-27 cytokines, which lead to a Th1 response. Infect. Immun. 74, 1480-1489 (2006).

32. Amsen, D. et al. Instruction of distinct CD4T helper cell fates by different notch ligands on antigen-presenting cells. Cell 117, 515-526 (2004).

33. Smit, J.J., Rudd, B.D. \& Lukacs, N.W. Plasmacytoid dendritic cells inhibit pulmonary immunopathology and promote clearance of respiratory syncytial virus. J. Exp. Med. 203, 1153-1159 (2006).

34. Blasius, A.L., Cella, M., Maldonado, J., Takai, T. \& Colonna, M. Siglec-H is an IPC-specific receptor that modulates type I IFN secretion through DAP12. Blood 107, 2474-2476 (2006).
35. Laouar, Y., Welte, T., Fu, X.Y. \& Flavell, R.A. STAT3 is required for Flt3L-dependent dendritic cell differentiation. Immunity 19, 903-912 (2003).

36. McGee, H.S., Edwan, J.H. \& Agrawal, D.K. Flt3-L Increases CD4+CD25+Foxp3+ICOS+ cells in the lung of cockroach-sensitized and challenged mice. Am J Respir Cell Mol Biol (2009); (e-pub ahead of print).

37. LoPiccolo, J., Blumenthal, G.M., Bernstein, W.B. \& Dennis, P.A. Targeting the PI3K/Akt/mTOR pathway: effective combinations and clinical considerations. Drug Resist Updat 11, 32-50 (2008).

38. Ruiter, G.A., Zerp, S.F., Bartelink, H., van Blitterswijk, W.J. \& Verheij, M. Alkyl-lysophospholipids activate the SAPK/JNK pathway and enhance radiation-induced apoptosis. Cancer Res 59, 2457-2463 (1999).

39. Behlke, M.A. Progress towards in vivo use of siRNAs. Mol. Ther. 13, 644-670 (2006).

40. Brazil, D.P., Yang, Z.Z. \& Hemmings, B.A. Advances in protein kinase B signalling: AKTion on multiple fronts. Trends Biochem. Sci. 29, 233-242 (2004).

41. Moretti, S. et al. The contribution of PARs to inflammation and immunity to fungi. Mucosal. Immunol. 1, 156-168 (2008).

42. Bozza, S. et al. Lack of Toll IL-1R8 exacerbates Th17 cell responses in fungal infection. J. Immunol. 180, 4022-4031 (2008).

43. Delgoffe, G.M. et al. The mTOR kinase differentially regulates effector and regulatory T cell lineage commitment. Immunity 30, 832-844 (2009).

44. Haxhinasto, S., Mathis, D. \& Benoist, C. The AKT-mTOR axis regulates de novo differentiation of CD4+Foxp3+ cells. J. Exp. Med. 205, 565-574 (2008).

45. Sauer, S. et al. T cell receptor signaling controls Foxp3 expression via PI3K, Akt, and mTOR. Proc. Natl. Acad. Sci. USA 105, 7797-7802 (2008).

46. Rivera, A. et al. Innate immune activation and CD4+T cell priming during respiratory fungal infection. Immunity $25,665-675$ (2006).

47. Ramaprakash, H., Ito, T., Standiford, T.J., Kunkel, S.L. \& Hogaboam, C.M. Toll-like receptor 9 modulates immune responses to Aspergillus fumigatus conidia in immunodeficient and allergic mice. Infect. Immun. 77, 108-119 (2009).

48. Hohl, T.M. et al. Aspergillus fumigatus triggers inflammatory responses by stage-specific beta-glucan display. PLoS Pathog 1, e30 (2005).

49. Gringhuis, S.I. et al. Dectin-1 directs T helper cell differentiation by controlling noncanonical NF-kappaB activation through Raf-1 and Syk. Nat. Immunol. 10, 203-213 (2009).

50. Beurel, E. \& Jope, R.S. Differential regulation of STAT family members by glycogen synthase kinase-3. J. Biol. Chem. 283, 21934-21944 (2008).

51. Bitko, V. \& Barik, S. Nasal delivery of siRNA. Methods Mol. Biol. 442, 75-82 (2008).

52. Zhang, W. et al. Inhibition of respiratory syncytial virus infection with intranasal siRNA nanoparticles targeting the viral NS1 gene. Nat. Med. 11, 56-62 (2005).

53. Gardy, J.L., Lynn, D.J., Brinkman, F.S. \& Hancock, R.E. Enabling a systems biology approach to immunology: focus on innate immunity. Trends Immunol. 30, 249-262 (2009).

54. Lebeis, S.L. \& Kalman, D. Aligning antimicrobial drug discovery with complex and redundant host-pathogen interactions. Cell Host Microbe 5 , 114-122 (2009).

55. Aimanianda, V. et al. Surface hydrophobin prevents immune recognition of airborne fungal spores. Nature 460, 1117-1121 (2009).

56. Asselin-Paturel, C., Brizard, G., Pin, J.J., Briere, F. \& Trinchieri, G. Mouse strain differences in plasmacytoid dendritic cell frequency and function revealed by a novel monoclonal antibody. J. Immunol. 171, 6466-6477 (2003).

57. Stephens-Romero, S.D., Mednick, A.J. \& Feldmesser, M. The pathogenesis of fatal outcome in murine pulmonary aspergillosis depends on the neutrophil depletion strategy. Infect. Immun. 73, 114-125 (2005).

58. Zhang, X. et al. Small interfering RNA targeting heme oxygenase-1 enhances ischemia-reperfusion-induced lung apoptosis. J. Biol. Chem. 279, 10677-10684 (2004). 